\title{
SERVICE NETWORK DESIGN IN SHORT AND LOCAL FRESH FOOD SUPPLY CHAIN*
}

\author{
Maxime OGier $^{1}$, Van-Dat Cung $^{1}$ And Julien Boissière ${ }^{2}$
}

\begin{abstract}
This paper aims at developing efficient solving methods for an original service network design problem imbued with sustainable issues. Indeed the network has to be designed for short and local supply chain and for fresh food products. The original features of the problem are the seasonality of supply, the limitation of transshipments for a product and no possibility of storage between consecutive periods. Decisions at strategic and tactical level are (1) decisions on a subset of hubs to open among a given set of potential locations, (2) transportation services to open between the actors and (3) flow quantities for the fresh food products. We propose for this problem a Mixed Integer Programming formulation and two solving techniques: Benders Decomposition and Dynamic Slope Scaling Procedure. These techniques are adapted to the problem and some experimental tests are conducted in order to compare the approaches on large-scale instances.
\end{abstract}

Keywords. Service network design, transshipment, multi-commodity flow, short supply chain, benders decomposition, Dynamic slope scaling procedure.

Mathematics Subject Classification. 90B10.

Received October 7, 2013. Accepted October 15, 2013.

* Funding for this research was provided by a grant from la Région Rhône-Alpes, Conseil Général de l'Isère, Chambre d'Agriculture de l'Isère.

1 Grenoble-INP/UJF-Grenoble 1/CNRS, G-SCOP UMR5272 Grenoble, 38031, France. maxime.ogier@grenoble-inp.fr; van-dat.cung@grenoble-inp.fr

2 Université de Savoie, LISTIC EA3703 Annecy 74016, France.

julien.boissiere@univ-savoie.fr 


\section{INTRODUCTION}

One of the major problems faced by French agriculture is the shortfall of the farmer's incomes. Over the last decades, farmers have been encouraged to produce more, while their unit selling price was decreasing. Nowadays in many regions in France coexist (1) supplies with medium-sized farms where various products of quality (freshness) are cultivated and (2) demands within important consumption area and a strong desire for product quality and traceability. Hence the idea has emerged to locally connect these supplies and demands. It is referred as short (and/or) local food supply chain. The main purpose of this kind of supply chain is to capture more end-use value for the farmers.

Short food supply chain is officially defined by the French Ministry of Agriculture as a marketing mode for agricultural products either through direct sales from producers to consumers, either through indirect sales, provided that there is only one middleman. In local food supply chain there may be more than one middleman but economic actors have to remain located on a restricted area. This paper deals with short and local fresh food supply chain, which involves notions of both relational and spatial proximity.

Short and local fresh food supply chain is linked to sustainable development. It should enable the development of an economy that cannot be relocated, a strengthening of social link between farmers and consumers, jobs creation for young farmers, conservation and transmission of know-how in a region, spatial planning, resistance to uncertainties thanks to diverse medium-sized structures, fresh and better quality products, and hopefully better health for consumers. However, the environmental impact must be put into perspective because of transport emissions [14].

Because of fewer middlemen in the supply chain, farmers have to take charge of a larger part of their products marketing and distribution, which is not their core business. It is feasible for direct sales, but for indirect sales (foodservice, supermarket) volumes are more important so the supply chain network has to be properly designed in order to organize products flows and to minimize transportation costs (in order to be competitive with global supply).

In this paper we are interested in service network design for short and local food supply chain (SND-SLSC). This is an optimization problem at both strategic and tactical level. The aim is (1) to find a subset of hub to open among a given set, (2) to set-up transportation services and (3) to determine flow quantities for the products in order to satisfy demands with a minimal transportation cost. This paper adopts the point of view of a local association or a political institution as final decision-maker who wants to evaluate different scenarios of the whole logistics system. This differs from the one of a profitable business actor who may want to optimize only direct and indirect sales. Section 1 presents a literature review on service network design. Then SND-SLSC problem is described and a Mixed Integer Program (MIP) formulation is given in Section 2. Section 3 details solving methods and experimental results are analyzed in Section 4. Conclusion and some prospects are drawn in the final section. 


\section{Literature REVIEW}

Service network design problem (SNDP) has been extensively studied in the literature. One may refer to $[2,5,15,16]$ for a survey. We are interested in traffic service network and not in transport service network (e.g. for bus or train). The main characteristic of NDP is to balance fixed cost for the design of the network and variable costs for the use of the network. Based on [6] the problem can be defined on a graph $\mathrm{G}=(\mathrm{N}, \mathrm{A})$ where $\mathrm{N}$ is a set of nodes and $\mathrm{A}$ a set of arcs connecting nodes. $\mathrm{N}$ can be separated in 3 (non-disjoint) sets: (1) origin, (2) destination, (3) transshipment of products. A capacity, a fixed cost (for design) and a linear cost (for utilization) can be associated with arcs. It is also possible to associate a fixed cost to nodes (location problem). The aim of NDP is to choose arcs to open in order to satisfy the demand for the destination nodes, at the lowest cost (sum of design and utilization costs).

NDP covers a wide range of problems: locations problems, multi-commodity capacitated network design problems (MCNDP), network flow problems (NFP). Our interest is not on MCNDP since it considers that origin and destinations are known for each commodity [7]. For the SND-SLSC problem, assignment of farmers and clients is not an entry data. Our interest is more on the coupled location problem and NFP. The aim of location problem is to choose, among a set of locations, which should be open and which clients they deliver. A review on the subject can be found in [13], and [11] present a study on NDP with capacitated facility location. In SND-SLSC hub locations for transshipment are considered to be different of farmers and clients locations since they cannot belong to one of these actor categories. Moreover in this study opening costs and capacities are not considered. Thus we are interested in a location problem close to a p-median problem which is known to be NP-hard [10]. Once hub location is fixed, there still remains a special NFP with fixed costs associated with opened arcs: Fixed Charge Network Flow Problem (FCNFP). The FCNFP is to select a set of arcs to open in the graph $\mathrm{G}$ and to find a feasible flow (to satisfy demands) in the resulting graph in order to minimize the sum of fixed and variables costs. This problem is also known to be NP-Hard [8]. Since this problem is difficult to solve, different techniques have been studied in order to solve the problem. See $[4,9,12]$ for different approaches.

In SND-SLSC, multi-period has to be considered since products seasonality induce regular changes in the available products, while the transshipment locations need to be the same for the whole set of periods. Moreover, local supply chains induce that the complete graph is not considered: if actors are too far from each other a direct link cannot exist. Short supply chain also induces that a product cannot flow through too many transshipment locations. 


\section{Problem formulation}

\subsection{DATA AND OBJeCtive}

The following data are considered for SND-SLSC:

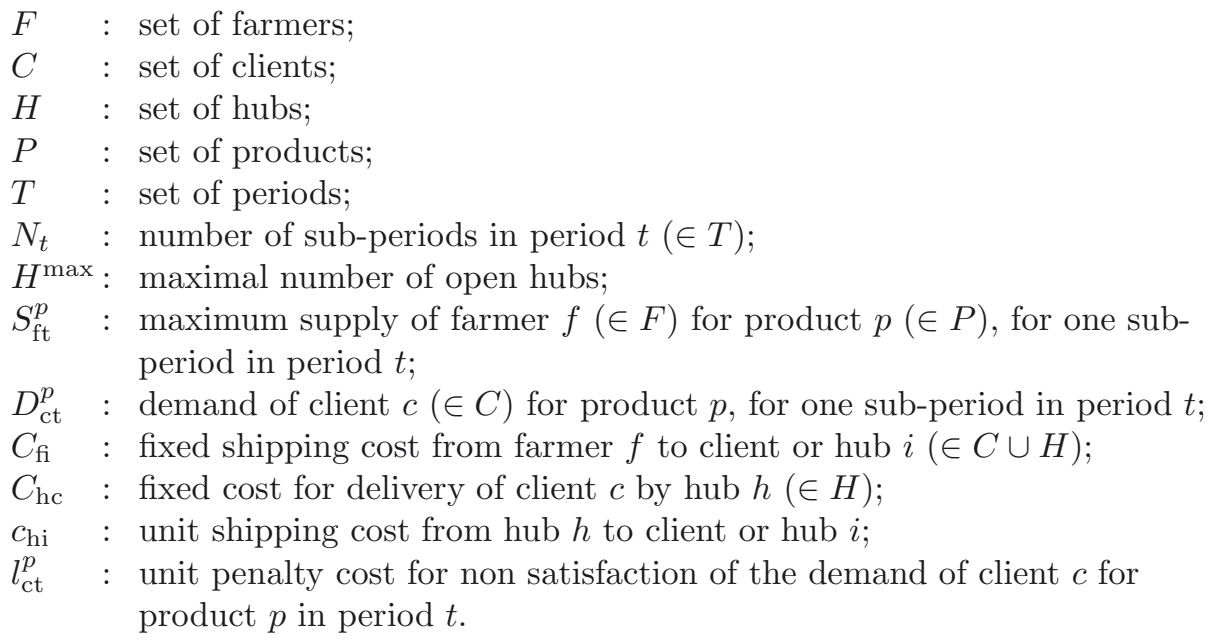

The considered logistic network contains 3 (disjoint) sets of actors. Farmers $(f \in F)$ cultivating a set of products $(p \in P)$ and who take charge of their products delivery. They can deliver clients $(c \in C)$ directly or logistics middlemen $(h \in H)$ who manage hubs. Direct delivery is considered since it is the core idea of short supply chains, and it permits to develop direct relationships between suppliers and clients. However, in order to satisfy all the demands with viable logistics costs and reasonable shipping distances for farmers, delivery through hubs with cross-dock operations is also considered. A restriction on the number of transshipments is added to take into account the features of short fresh food supply chain.

Moreover, since all the actors are located on a restricted area, their proximity permits to facilitate collaborations, to establish long term relationships, to share common objectives and to foster some local associations or political entities acting as central coordinator of the network. Hence our proposed model assumes a centralized decision maker who is in charge of coordinating the supply and demand, and managing the set of hubs. The model allows to evaluate the logistics costs of the whole system.

Besides, because of production seasonality, a set of periods $(t \in T)$ is considered. To evaluate the transportation costs, a further division of the periods is made. For each period $t, N_{t}$ sub-periods are considered with the same supply of farmers $\left(S_{\mathrm{ft}}^{p}\right)$ and demand of clients $\left(D_{\mathrm{ct}}^{p}\right)$. The transportation plan is repeated for the $N_{t}$ subperiods of each period $t$, leading to multiply transportation costs by $N_{t}$. Products are fresh, so they cannot be stored between two periods or two sub-periods. As an 
example, each period $t$ can represent a month since seasonality is different from one month to another; sub-periods can represent a week if clients want to be weekly delivered.

The aim is to determine among a set of potential hubs which ones should be opened in order to minimize logistics costs. A maximal number of open hubs $\left(H^{\max }\right)$ is considered, and this parameter allows to evaluate different investment scenarios for the central decision-maker. The interest is mainly on routing costs, as opening costs for hubs are not considered in this work.

For the central decision-maker, opening costs are difficult to evaluate since they highly depend on the quantity of products transshipping through the hub. For example, with a small quantity, one refrigerated truck can play the role of hub, while with an important quantity a real cross-docking platform should be built.

Farmers deliver clients or hubs with small vehicles so the shipping costs $\left(C_{\mathrm{fc}}\right.$, $\left.C_{\text {fh }}\right)$ are dependent on the distance from the farmer to the client/hub, and independent of the transported quantity. It is considered the loading quantity has no influence on the cost for a small vehicle. Trucks leave the hubs to deliver other hubs or clients $(i \in H \cup C)$ : the shipping cost $\left(c_{\mathrm{hi}}\right)$ is dependent of the transported quantity because it is done through a regular service, and independent of the distance. This shipping cost can include hub operating costs. A fixed cost $\left(C_{\mathrm{hc}}\right)$ is also incurred when a client is delivered by a hub. This fixed cost represents the time for parking, unloading the truck and the delivery of the client. When a hub delivers another hub, this fixed cost is not considered since it is assumed to be easier and faster to deliver a hub. Hence, the model contains pure fixed cost arcs (from farmers to other actors), pure linear cost arcs (between hubs) and concave costs arcs (from hubs to clients). Over the planning horizon, the costs are considered constant.

Moreover, if demand is not satisfied, there is a unit penalty cost $l_{\mathrm{ct}}^{p}$ to pay. This permits to consider total supply can be lower than total demand. In this case a penalty cost greater than the maximum transportation cost as follows,

$$
l_{\mathrm{ct}}^{p}>\max \left\{\max _{f \in F}\left\{C_{\mathrm{fc}}\right\} ; \max _{h, h^{\prime} \in H}\left\{\max _{f \in F}\left\{C_{\mathrm{fh}}\right\}+C_{h^{\prime} c}+c_{\mathrm{hh}^{\prime}}+c_{h^{\prime} c}\right\}\right\}
$$

can be considered in order to ensure the best satisfaction of the demand because it is costless to deliver a demand than to pay a penalty. A lower value of the penalty cost can be considered. It would bound the maximum cost to pay for transportation of one unit of the demand. A demand could thus be deliberately unsatisfied because of a too high transportation cost.

Notice that since the considered supply chain is local, it is possible to limit farmers and hubs scope. It suffices not to consider the variables related to service and flow between actors $i$ and $j$, in a pre-processing stage, if distance between these two actors is too large. 


\subsection{RESTRICTION ON THE NUMBER OF TRANSSHIPMENTS}

Models for NDP have been extensively studied $[1,6]$. But the modelling of our problem is more complex because we have to take into account simultaneously the fixed and variable costs in the objective function, and the restriction to two maximal transshipments for one product. Indeed, an arc-based formulation can easily take into account the costs structure, but restriction on transshipments is difficult to express, while a path-based formulation is well-adapted for this last point but not for the first.

This work proposes an arc-based formulation with the restriction on transshipments. The idea is to double each vertex of the graph that represents a hub. One vertex represents the input part of the hub (i.e. the place where come products from farmers) while the other vertex represents the output part (i.e. the place from which products flow to clients). For each product an arc is added to represent the product flow between the two vertices (the two parts of the hub). This permits to ensure that a product flow coming from another hubs reaches the output part of the hub while a flow going to another hub leaves from the input part. Thus it becomes impossible for a product to be transshipped more than twice. An example is given in Figure 1, for one hub represented by the vertex $h_{2}$ and changed by vertices $h_{2}^{\prime}$ and $h_{2}^{\prime \prime}$. For the sake of simplicity this example deals with one product and variables $x$ represent flow quantities associated with each arc. The new variable $x_{p c}$ represents the quantity coming from farmers and directly leaving to clients (without transshipment by another hub). The associated cost is 0. Regarding the formulation, the first approach is to use two flow balance constraints with the new variable $\left(x_{p c}\right)$. For the example in Figure 1 with focus on hub $h_{2}$ these two constraints are:

$$
\begin{aligned}
& x_{p_{2}}=x_{p c_{2}}+x_{h_{2}}, \\
& x_{h_{1}}+x_{p c_{2}}=x_{c_{2}} .
\end{aligned}
$$

Instead of adding new variables and constraints (3.2) and (3.3) (plus non-negativity of the variables), the flow balance constraint in $h_{2}$ can hold:

$$
x_{h_{1}}+x_{p_{2}}=x_{h_{2}}+x_{c_{2}} \text {. }
$$

and the two following constraints can be added:

$$
\begin{aligned}
& x_{h_{1}} \leq x_{c_{2}}, \\
& x_{h_{2}} \leq x_{p_{2}} .
\end{aligned}
$$



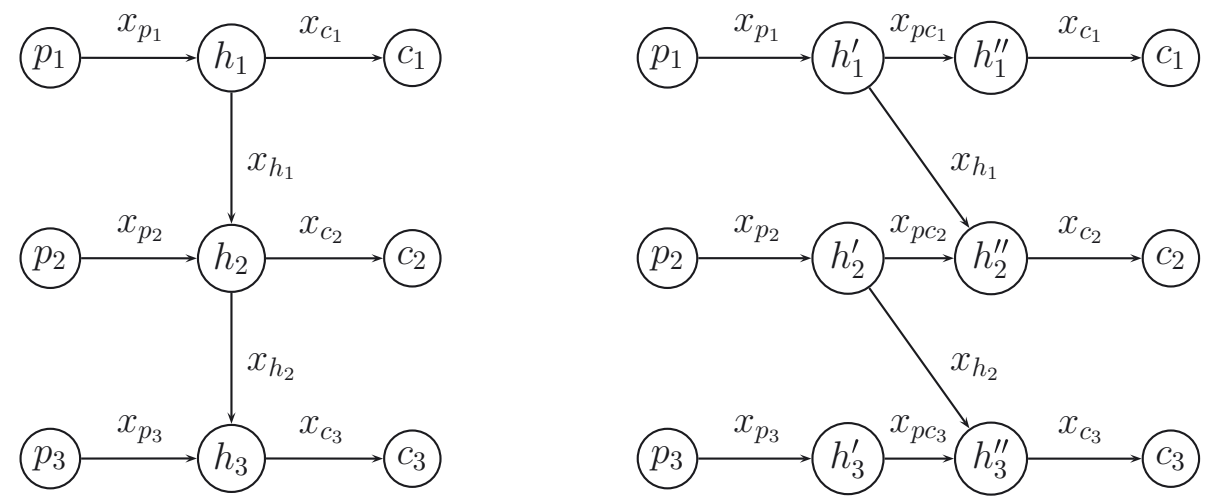

FiguRE 1. Restriction on the number of transshipments with an arc-based model.

\subsection{Mixed Integer Programming Formulation}

The proposed formulation comes from the arc-based formulation of the problem. The decision variables are as follows:

$x_{\text {fit }}^{p} \quad$ : quantities for each product $p(\in P)$ which flow in each sub-period of period $t(\in T)$, between farmer $f(\in F)$ and client or hub $i(\in C \cup H)$;

$x_{\text {hit }}^{p}:$ quantities for each product $p$ which flow in each sub-period of period $t$, between hub $h(\in H)$ and client or hub $i$;

$z_{\mathrm{ct}}^{p} \quad$ : shortage in the demand of client $c(\in C)$ for product $p$ in each sub-period of period $t$;

$y_{\text {fit }}:\left\{\begin{array}{l}1 \text { if farmer } f \text { delivers client or hub } i \text { in period } t, \\ 0 \text { otherwise; }\end{array}\right.$

$y_{\text {hct }}:\left\{\begin{array}{l}1 \text { if hub } h \text { delivers client } c \text { in period } t \\ 0 \text { otherwise; }\end{array}\right.$

$y_{h}:\left\{\begin{array}{l}1 \text { if hub } h \text { is open, } \\ 0 \text { otherwise. }\end{array}\right.$

The Mixed Integer Programming (MIP) is the following.

$$
\begin{aligned}
\operatorname{Min} & \sum_{t \in T} N_{t} \cdot\left(\sum_{f \in F, i \in C \cup H} C_{\mathrm{fi}} \cdot y_{\mathrm{fit}}+\sum_{h \in H, c \in C} C_{\mathrm{hc}} \cdot y_{\mathrm{hct}}\right. \\
& \left.+\sum_{h \in H, i \in C \cup H, p \in P} c_{\mathrm{hi}} \cdot x_{\mathrm{hit}}^{p}+\sum_{c \in C, p \in P} l_{\mathrm{ct}}^{p} \cdot z_{\mathrm{ct}}^{p}\right)
\end{aligned}
$$

s.t. 


$$
\begin{aligned}
& \sum_{h \in H} y_{h} \leq H^{\max } \\
& \sum_{i \in C \cup H} x_{\mathrm{fit}}^{p} \leq S_{\mathrm{ft}}^{p} \\
& \forall f \in F, p \in P, t \in T \text {; } \\
& x_{\mathrm{fit}}^{p} \leq S_{\mathrm{ft}}^{p} \cdot y_{\mathrm{fit}} \\
& \forall f \in F, i \in C \cup H, p \in P, t \in T ; \\
& \sum_{j \in F \cup H} x_{\mathrm{jct}}^{p}+z_{\mathrm{ct}}^{p}=D_{\mathrm{ct}}^{p} \\
& x_{\mathrm{hct}}^{p} \leq D_{\mathrm{ct}}^{p} \cdot y_{\mathrm{hct}} \\
& \sum_{j \in F \cup H} x_{\mathrm{jht}}^{p}=\sum_{i \in C \cup H} x_{\mathrm{hit}}^{p} \\
& x_{\mathrm{fht}}^{p} \leq S_{\mathrm{ft}}^{p} \cdot y_{h} \\
& x_{\mathrm{hh}^{\prime} \mathrm{t}}^{p} \leq \min \left\{\sum_{f \in F} S_{\mathrm{ft}}^{p} ; \sum_{c \in C} D_{\mathrm{ct}}^{p}\right\} \cdot y_{h} \quad \forall h, h^{\prime} \in H, p \in P, t \in T ; \\
& \sum_{h^{\prime} \in H} x_{\mathrm{h}^{\prime} \mathrm{ht}}^{p} \leq \sum_{c \in C} x_{\mathrm{hct}}^{p} \\
& \forall h \in H, p \in P, t \in T \text {; } \\
& \sum_{h^{\prime} \in H} x_{\mathrm{hh}^{\prime} \mathrm{t}}^{p} \leq \sum_{f \in F} x_{\mathrm{fht}}^{p} \\
& \forall h \in H, p \in P, t \in T \text {; } \\
& y_{h}, y_{\text {fit }}, y_{\text {hct }} \in\{0 ; 1\} \\
& x_{\mathrm{ijt}}^{p}, z_{\mathrm{ct}}^{p} \geq 0 \\
& \forall h \in H, i \in C \cup H, c \in C, t \in T \text {; } \\
& \forall i \in F \cup H, j \in H \cup C, c \in C, p \in P, t \in T \text {. }
\end{aligned}
$$

The objective function (3.7) is to minimize fixed transportation costs for farmers and hubs, linear transportation costs for hubs and shortage costs. For each period $t$, costs are multiplied by the number of sub-periods $N_{t}$ where the distribution scheme is repeated. Constraint (3.8) is the upper bound on the number of hubs to open. Constraints (3.9) ensure the respect of supply quantities for the farmers. Constraints (3.11) and (3.13) are flow balance constraints respectively for clients and hubs. Constraints (3.10) and (3.12) are linking constraints for the opening of transportation services. Constraints (3.14) and (3.15) are linking constraints for the opening of hubs. Constraints (3.16) and (3.17) ensure a product is transshipped at most twice.

Notice that this model can be extended by considering a fixed opening cost $f_{h}$ for hub $h$. In order to do so, the term $\sum_{h \in H} f_{h} \cdot y_{h}$ should be added in the objective function (3.7), and the constraint (3.8) should be deleted.

\section{Solving TECHNIQUeS}

\subsection{BRANCH \& Cut}

Since the problem has a MIP formulation, it appears natural to solve it with Branch \& Cut (B\&C) techniques. Indeed, cuts are generated during the Branch 
\& Bound in order to speed up the solving time. These techniques calculate lower bounds $\left(z_{\mathrm{LB}}\right)$ and upper bounds $\left(z_{\mathrm{UB}}\right)$ of the optimal value $z^{*}$. This permits to reach an optimal solution, or at least to find a solution with a guaranty on the gap with an optimal solution $\left(\frac{z_{\mathrm{UB}}-z_{\mathrm{LB}}}{z_{\mathrm{LB}}}\right)$. Commercial solver CPLEX 12.2 is used to solve the problem with these techniques. For a more detailed study of cut generation for fixed charge network flow problem, the reader can refer to [12]. The use of commercial solver however does not allow using the characteristics of the SND-SLSC problem. A specific B\&C for this problem could be implemented, but it goes beyond the purpose of this study.

\subsection{Benders Decomposition}

Benders Decomposition [3] is a mathematical technique for solving difficult problems, and more specifically problems formulated as MIP. The initial problem has the following formulation:

$$
\begin{array}{cl}
\operatorname{Min} & c^{T} \cdot x+f(y) ; \\
\text { s.t. } & A \cdot x+g(y) \geq b ; \\
& D \cdot y \geq e ; \\
& y \in \mathbb{N} ; \\
& x \geq 0 .
\end{array}
$$

Benders Decomposition consists in decomposing the problem with a master problem and a sub-problem. The master problem captures the integer (or boolean) variables (also called design variables for NDP) plus one floating variable. The subproblem is formulated as a Linear Program (LP), i.e. with only floating variables, considering $\bar{y}$ the solution of the master problem as a data:

$$
\begin{array}{cl}
\operatorname{Min} & c^{T} \cdot x+f(\bar{y}) \\
\text { s.t. } & A \cdot x \geq b-g(\bar{y}) ; \\
& x \geq 0 .
\end{array}
$$

The sub-problem is easier to solve than the original problem. Note that depending on the design variables chosen $(\bar{y})$ it can be infeasible. But this situation cannot happen in SND-SLSC since demand shortages are in the model. The dual formulation of the sub-problem is used because it permits to solve the problem with constraints independent of the design variables. It is formulated as follows:

$$
\begin{aligned}
\operatorname{Max} & \beta=u \cdot(b-g(\bar{y}))+f(\bar{y}) \\
\text { s.t. } & u \cdot A \geq c \\
& u \geq 0
\end{aligned}
$$


The solution of this dual sub-problem $\left(\beta^{*}\right)$ is a lower bound for the problem with variables $\bar{y}$. The dual solution $\left(u^{*}\right)$ is finite, so it is possible to add this bound in the master problem so that it is valid for any value of the design variables $(y)$ and not only for the fixed values $(\bar{y})$. Indeed, the constraints of the dual sub-problem are independent of $y$ so the solution $u^{*}$ remains a feasible solution for any value of $y$.

Hence a Benders cut is added in the master problem, which is formulated as:

$$
\begin{aligned}
\text { Min } & z \\
\text { s.t. } & z \geq u^{*} \cdot(b-g(y))+f(y) \\
& z \geq 0 ;(4.3)-(4.4)
\end{aligned}
$$

The solution of this master problem gives a lower bound for the initial problem. The new integer solutions $(y)$ permit to solve a new sub-problem and to iterate the method. Each sub-problem generates a cut in the master problem which is the following after $k$ iterations:

$$
\begin{aligned}
\text { Min } & z ; \\
\text { s.t. } & z \geq u^{i} \cdot(b-g(y))+f(y) \quad \forall i=1 \ldots k ;(4.14) .
\end{aligned}
$$

In Benders Decomposition, cuts are iteratively added in the master problem until the difference between the lower bound given by the master problem and the upper bound given by the sub-problem are under a certain fixed $\varepsilon$. Benders Decomposition is considered among the "most successful solution approaches" for network design problem with fixed costs [4].

This approach has been applied to SND-SLSC problem. One of the advantages is that the sub-problem can be decomposed into several smaller sub-problems since when the design variables are fixed $\left(y_{h}, y_{f c t}, y_{\text {fht }}, y_{\text {hct }}\right)$, the flow problem can be regarded separately for each period and for each product. Moreover, other constraints have been integrated in the master problem in order to avoid poor quality solutions in the sub-problem.

$$
\begin{array}{ll}
\sum_{f \in F} y_{\mathrm{fhm}} \leq|F| \cdot y_{h} & \forall h \in H, t \in T \\
\sum_{c \in C} y_{\mathrm{fcm}} \leq|C| \cdot y_{h} & \forall h \in H, t \in T
\end{array}
$$

Constraints (4.17) and (4.18) ensure a service between a farmer (or client) and a hub is opened only if the hub is open. Moreover, if not enough services are opened in the master problem, only a few part of the demand can be satisfied in the subproblems, hence there is a huge shortage penalty cost. These constraints in the master problem permit not to spend time on very suboptimal solutions. Moreover, the advantage of Benders Decomposition is that the hub location problem can be handled directly in the master problem; and the master problem gives a lower 
bound for the problem so for each solution there is a guaranty on the gap with an optimal solution.

This method has also the advantage to be general, and fixed opening costs $f_{h}$ for hubs can be easily considered. The master problem should be modified adding the term $\sum_{h \in H} f_{h} \cdot y_{h}$ in the objective function and removing the constraint (3.8).

\subsection{Dynamic SLope scaling Procedure (DSSP)}

DSSP is an original technique first proposed in [9] in order to solve FCNFP. The objective is to decrease solving time and memory usage for large scale instances of the problem. The basic idea is to iteratively solve Linear Programs (LP), updating the objective function without changing constraints. The FCNFP studied in [9] is formulated as follows:

$$
\begin{aligned}
& \operatorname{Min} f(x)=\sum_{j=1}^{n} f_{j}\left(x_{j}\right) \text {; } \\
& \text { s.t. } A \cdot x=b \text {; } \\
& 0 \leq x \leq u \\
& \text { where } f_{j}\left(x_{j}\right)= \begin{cases}0 & \text { if } x_{j}=0 ; \\
s_{j}+c_{j} \cdot x_{j} ; s_{j} \geq 0 & \text { if } x_{j}>0 .\end{cases}
\end{aligned}
$$

$s_{j}$ is the fixed cost associated with the opening of the arc $j$ and $c_{j}$ is the unit cost associated with the level of activity of the arc $j$.

The idea is to find a linear factor which integrates the fixed and unit costs. The objective function for each vector $\hat{x}$ is rewritten as:

$$
\begin{gathered}
\bar{f}(\hat{x})=\sum_{j=1}^{n} \bar{f}_{j}\left(\hat{x}_{j}\right)=\sum_{j=1}^{n} \bar{c}_{j}\left(\hat{x}_{j}\right) \cdot \hat{x}_{j} ; \\
\text { where } \bar{c}_{j}\left(\hat{x}_{j}\right)= \begin{cases}M & \text { if } \hat{x}_{j}=0 ; \\
c_{j}+\frac{s_{j}}{\hat{x}_{j}} & \text { if } \hat{x}_{j}>0 ;\end{cases}
\end{gathered}
$$

where $M$ is a large number.

This reformulation considers $\bar{c}_{j}\left(\hat{x}_{j}\right)$ is the slope between the origin point $(0 ; 0)$ and the point $\left(\hat{x}_{j} ; \bar{f}_{j}\left(\hat{x}_{j}\right)\right)$ like shown in Figure 2 . The aim of the reformulation is to find a LP formulation with the same optimal solution than the original problem. Hence costs have to be updated at each iteration. Once the solution $\bar{x}^{k}$ of iteration $k$ is obtained, costs for iteration $k+1$ are defined as follows:

$$
\bar{c}_{j}\left(\bar{x}_{j}\right)^{k+1}= \begin{cases}c_{j}+\frac{s_{j}}{\bar{x}_{j}^{k}} & \text { if } \bar{x}_{j}^{k}>0 ; \\ \bar{c}_{j}^{k} & \text { if } \bar{x}_{j}^{k}=0 .\end{cases}
$$

The procedure ends when the solutions of two consecutive iterations of the LP problem are the same $\left(\bar{x}_{j}^{k-1}=\bar{x}_{j}^{k} \forall j\right)$. It is also possible to define a maximal 


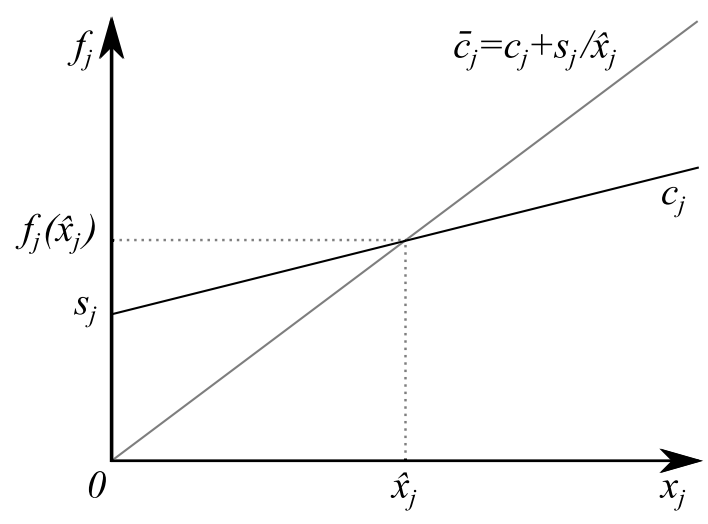

Figure 2. Slope for the point $\left(\hat{x}_{j} ; \bar{f}_{j}\left(\hat{x}_{j}\right)\right)[9]$.

number of iteration and then to use a heuristic procedure in order to obtain a feasible solution for the original problem. However, DSSP do not guarantee the solution found is the optimal solution. But results of [9] indicate this method can give rather good results.

DSSP needs to be adapted to the SND-SLSC problem for two reasons: (1) decisions for hub location cannot be considered since there are no associated costs, (2) the network flow is multi-commodity. We consider that if the number of potential hub location is not too big, it is possible to test the different combinations. The number of combinations is $\left(\begin{array}{c}|H| \\ H^{\max }\end{array}\right)$. In other cases, it should be possible to use a heuristic procedure in order to first define a set of good solutions for the hub opening (based on a p-median formulation), and then to apply DSSP for each element of this solution set. Since SND-SLSC is multi-commodity, initial slopes are defined as follows.

$$
\begin{aligned}
\bar{c}_{\mathrm{fipt}}\left(\bar{x}_{\mathrm{fit}}^{p}\right)^{0}= & \left\{\begin{array}{rl}
0 & \text { if } S_{\mathrm{ft}}^{p}=0 ; \\
\frac{C_{\mathrm{fi}}}{\sum_{p^{\prime} \in P} S_{\mathrm{ft}}^{p^{\prime}}} & \text { if } S_{\mathrm{ft}}^{p}>0 ;
\end{array} \quad \forall f \in F, i \in C \cup H, p \in P, t \in T .\right. \\
& \bar{c}_{\mathrm{hipt}}\left(\bar{x}_{\mathrm{hit}}^{p}\right)^{0}=c_{\mathrm{hi}} \quad \forall h \in H, i \in C \cup H, p \in P, t \in T .
\end{aligned}
$$

The updating scheme is:

$$
\begin{gathered}
\bar{c}_{\mathrm{fipt}}\left(\bar{x}_{\mathrm{fit}}^{p}\right)^{k+1}= \begin{cases}\frac{C_{\mathrm{fi}}^{p}}{\sum_{p^{\prime} \in P}\left(\bar{x}_{\mathrm{fit}}^{p^{\prime}}\right)^{k}} & \text { if }\left(\bar{x}_{\mathrm{fit}}^{p}\right)^{k}>0 ; \\
\bar{c}_{\mathrm{fipt}}^{k} & \text { if }\left(\bar{x}_{\mathrm{fit}}^{p}\right)^{k}=0 ;\end{cases} \\
\forall f \in F, i \in C \cup H, p \in P, t \in T . \\
\bar{c}_{\mathrm{hcpt}}\left(\bar{x}_{\mathrm{hct}}^{p}\right)^{k+1}= \begin{cases}c_{\mathrm{hc}}+\frac{C_{\mathrm{hc}}^{p}}{\sum_{p^{\prime} \in P}\left(\bar{x}_{\mathrm{hct}}^{p}\right)^{k}} & \text { if }\left(\bar{x}_{\mathrm{hct}}^{p}\right)^{k}>0 ; \\
\bar{c}_{\mathrm{hcpt}}^{k} & \text { if }\left(\bar{x}_{\mathrm{hct}}^{p}\right)^{k}=0 ;\end{cases}
\end{gathered}
$$




$$
\begin{gathered}
\forall h \in H, c \in C, p \in P, t \in T . \\
\bar{c}_{\mathrm{hh}^{\prime} \mathrm{pt}}\left(\bar{x}_{\mathrm{hh}^{\prime} \mathrm{t}}^{p}\right)^{k+1}=c_{\mathrm{hh}^{\prime}} \quad \forall h, h^{\prime} \in H, p \in P, t \in T .
\end{gathered}
$$

Moreover, the LP formulation can be decomposed by period and by product in order to solve several smaller LP. This permits to solve large scale problems.

This method is specific to the proposed formulation of the problem. Hence fixed opening cost $f_{h}$ for hubs cannot be taken into consideration in a direct way. Indeed, it would necessitate to test all the values of $H^{\max } \in\{0 ; \ldots ;|H|\}$, and for each value to test the different combinations of open hubs, leading to $\sum_{k=0}^{|H|}\left(\begin{array}{c}|H| \\ k\end{array}\right)=2^{|H|}$ possibilities. For each possibility, the cost of open hubs is added to the flow cost calculated with DSSP. Even though DSSP is an heuristic method, this does not seem reasonable to generate all $2^{|H|}$ combinations. The previous idea proposed to solve a p-median problem (with the costs $f_{h}$ ) to generate a reduced set of potential solutions could be more interesting in this case.

\section{EXPERIMENTAL RESUltS}

Computational tests have been conducted in order to compare the methods exposed in Section 4. Two sets of instances are considered ${ }^{3}$. The first one (Set 1) permits to evaluate the methods on different sizes of instances with different geographical repartition of the actors. The second one (Set 2) contains instances with more hubs. This will allow to test the impact of the constraints on the maximum number of transshipments.

For Set 1, three sizes of instances are considered, and described in Table 1. We have considered that the set of products can be separated into two categories (of the same size): fruits and vegetables. One half of the farmers produce only fruits while the other half produce only vegetables. Since the study is on a local supply chain, clients and hubs are randomly located on a restricted area of $100 \mathrm{~km}$ by $50 \mathrm{~km}$. Spatial repartition of the farmers can also be randomly generated $(\mathrm{R})$ or clustered $(\mathrm{C})$ on sub-areas with high density. Moreover the spatial repartition of fruits farmers and vegetables farmers can be mixed $(\mathrm{M})$ or partitioned $(\mathrm{P})$, i.e. areas are dedicated to fruits or to vegetables. Hence 12 instances are considered, and named with three letters stating respectively the size, the spatial repartition of actors and the spatial repartition of fruits and vegetables farmers. For each instance it is assumed there are 4 deliveries a month $\left(N_{t}=4\right)$, and the the maximal number of hubs to open is $H^{\max }=2$. For each instance, assuming $U(a ; b)$ is the uniform distribution between $a$ and $b$, farmers supply (for fruits or vegetables) is $S_{\mathrm{ft}}^{p}=U(200 ; 300) \cdot s_{t}^{p}$ where $s_{t}^{p}$ represents the seasonality of product $p$ in period $t$ and is equal to 0 or 1 . Clients' demand is $D_{\text {ct }}^{p}=U(100 ; 150) \cdot s_{t}^{p}$. Since each product $p$ is produced by only a half of the farmers, supply and demands are equal

\footnotetext{
${ }^{3}$ Instances are available at http://www.g-scop.grenoble-inp.fr/recherche/resources-537901.kjsp
} 
TABLE 1. Instance sizes description for Set 1.

\begin{tabular}{lccccc}
\hline Instance type & $|F|$ & $|C|$ & $|H|$ & $|P|$ & $|T|$ \\
\hline Small (S) & 20 & 20 & 5 & 4 & 12 \\
Medium (M) & 50 & 50 & 5 & 10 & 12 \\
Large (L) & 50 & 100 & 5 & 16 & 12 \\
\hline
\end{tabular}

TABle 2. CPU time (in seconds) for Set 1.

\begin{tabular}{lcccc}
\hline Instance & $\begin{array}{c}\text { B\&C } \\
(\text { CPLEX 2\% })\end{array}$ & $\begin{array}{c}\text { B\&C } \\
(\text { CPLEX 5\%) }\end{array}$ & $\begin{array}{c}\text { Benders } \\
\text { decomposition }(5 \%)\end{array}$ & DSSP \\
\hline S-CP & 29.63 & 30.10 & 10544.27 & 81.55 \\
S-CM & 53.44 & 50.08 & 5.42 & 66.54 \\
S-RP & 31.28 & 34.78 & 3.55 & 71.77 \\
S-RM & 35.59 & 34.23 & 4.18 & 79.92 \\
M-CP & 1691.00 & 1757.76 & 11108.06 & 2406.87 \\
M-CM & 3084.05 & 3225.31 & 33.61 & 1972.07 \\
M-RP & 2033.59 & 1986.58 & 10806.20 & 2218.38 \\
M-RM & 904.07 & 919.77 & 162.17 & 2549.91 \\
L-CP & 3260.97 & 3312.11 & 185.17 & 7241.53 \\
L-CM & 6241.31 & 5801.11 & 293.97 & 6060.13 \\
L-RP & 5065.45 & 5025.09 & 107.51 & 7960.56 \\
L-RM & 5591.98 & 5383.32 & 76.58 & 6518.57 \\
\hline
\end{tabular}

in average. Let $d_{\mathrm{fi}}$ be the distance between farmer $f$ and client or hub $i$. Fixed costs for farmers are $C_{\mathrm{fi}}=0.8 \cdot 2 \cdot d_{\mathrm{fi}}$. For hubs, $C_{\mathrm{hc}}=4$ and $c_{\mathrm{hi}}=0.18$ and $l_{\mathrm{ct}}^{p}=10 \cdot D_{\mathrm{ct}}^{p}$. These costs (in Euro) are similar to the ones observed in field studies conducted by French local associations. The high value of the penalty costs makes satisfaction of the demands a key issue, especially concerning large clients. Moreover, a scope of action of $20 \mathrm{~km}$ is set for the direct shipments from farmers to clients. The variables $y_{f c t}$ and $x_{f c t}^{p}$ are defined in the model if and only if $d_{\mathrm{fc}} \leq 20$.

Benders Decomposition and DSSP have been implemented in Java using CPLEX 12.2 (callable library) for solving LP and MIP problems. The original problem has been modelled with OPL Studio and has been solved with CPLEX 12.2. The processor is an Intel Xeon $2.5 \mathrm{GHz}$ with 10 Gbytes of RAM. The stopping criterion for $\mathrm{B} \& \mathrm{C}$ with CPLEX is a gap $\left(\frac{z_{\mathrm{UB}}-z_{\mathrm{LB}}}{z_{\mathrm{LB}}}\right)$ less than $k \%$. Two gap values have been experimented: $2 \%$ and $5 \%$. The stopping criterion for Benders Decomposition is a gap less than $5 \%$ and a computational time less than 3 hours. Other tests not presented here have shown several hours of solving time for small instances when the gap for Benders Decomposition is $2 \%$. For DSSP there is no gap to calculate, so the stopping criterion is only the finding of a same solution for two consecutive iterations of the method. CPU time results are presented in Table 2.

The results show the very good performance of Benders Decomposition on solving time criterion, except on 3 instances (S-CP, M-CP, M-RP) where the gap of 
TABLE 3. Gap with CPLEX lower bound (in \%) for Set 1.

\begin{tabular}{lccc}
\hline Instance & $\begin{array}{r}\text { B\&C } \\
(\text { CPLEX 2\% \& 5\%) }\end{array}$ & $\begin{array}{c}\text { Benders } \\
\text { Decomposition }(5 \%)\end{array}$ & DSSP \\
\hline S-CP & 1.26 & 3.96 & 0.81 \\
S-CM & 0.90 & 2.45 & 0.49 \\
S-RP & 0.86 & 1.68 & 0.53 \\
S-RM & 1.08 & 2.67 & 0.56 \\
M-CP & 1.03 & 3.62 & 0.66 \\
M-CM & 1.13 & 2.85 & 0.53 \\
M-RP & 1.04 & 3.13 & 0.55 \\
M-RM & 1.41 & 4.10 & 0.62 \\
L-CP & 0.01 & 0.06 & 0.01 \\
L-CM & 0.02 & 2.30 & 0.01 \\
L-RP & 0.01 & 0.03 & 0.01 \\
L-RM & 0.02 & 0.97 & 0.01 \\
\hline
\end{tabular}

$5 \%$ has not been reached after 3 hours. The solving times for DSSP are often larger than solving times for B\&C with CPLEX. It can be noticed that for clustered and mixed farmers' instances (M-CM and L-CM), the solving times are lower for DSSP than for B\&C with a $2 \%$ gap. The performance of DSSP can be improved if all the combinations of open hubs are not solved. Indeed, for each instance, a DSSP problem is solved $\left(\begin{array}{c}|H| \\ H^{\max }\end{array}\right)=\left(\begin{array}{c}5 \\ 2\end{array}\right)=10$ times. A pre-processing based on a p-median problem could permit to only evaluate the most interesting subsets of hubs reducing the computational time. Though p-median problem is NP-hard, the instance considered are easier since we can omit products and periods. The B\&C method has been tested with two gap values, but the difference on the solving time is really small. Most of the computational time is spent in generating solutions with a very high gap, but at the end of the resolution, the $\mathrm{B} \& \mathrm{C}$ method uses to finish with solutions of gap less than $2 \%$ even if the stopping criterion is fixed at $5 \%$.

Performance results are presented in Table 3 for the gap with the lower bound given by CPLEX in the $\mathrm{B} \& \mathrm{C}$. The $\mathrm{B} \& \mathrm{C}$ with the two gap values gives the same results for lower and upper bounds. Hence only the $\mathrm{B} \& \mathrm{C}$ with a $2 \%$ gap is considered from here. For each method $m \in\{B \& C$; Benders; DSSP $\}$, the gap is $\frac{z_{\mathrm{UB}}^{m}-z_{\mathrm{LB}}^{B \& C}}{z_{\mathrm{LB}}^{B \& C}}$, where $z_{\mathrm{UB}}^{m}$ is the objective function of the solution of the method $m$. For the B\&C with CPLEX, the gap is under $2 \%$ since it is the stopping criterion.

These results show the very good quality of the solutions given by the DSSP method. Indeed, the gap is always lower than 1\%, and better than the gap of the B\&C with CPLEX. Though DSSP requires more computational time than B\&C, it gives better solutions. Moreover, Benders Decomposition gives quite good results, especially for large instances. More detailed results, with lower bounds of Benders Decomposition are presented in Table 7 of the Appendix. Compared to B\&C with CPLEX, Benders Decomposition has rather good lower bounds since they are close to the ones provided by CPLEX and the average gap between the lower bounds 
TABLE 4. Hubs parameters for Set 2.

\begin{tabular}{lcccccc}
\hline $\begin{array}{l}\text { Instance } \\
\text { name }\end{array}$ & $\mathrm{M}-\mathrm{CP}_{2}^{5}$ & $\mathrm{M}-\mathrm{CP}_{3}^{5}$ & $\mathrm{M}-\mathrm{CP}_{3}^{7}$ & $\mathrm{M}-\mathrm{CP}_{5}^{7}$ & $\mathrm{M}-\mathrm{CP}_{3}^{10}$ & $\mathrm{M}-\mathrm{CP}_{5}^{10}$ \\
\hline$|H|$ & 5 & 5 & 7 & 7 & 10 & 10 \\
$H^{\max }$ & 2 & 3 & 3 & 5 & 3 & 5 \\
\hline
\end{tabular}

$\frac{z_{\mathrm{LB}}^{B \text { enders }}-z_{\mathrm{LB}}^{B \& C}}{z_{\mathrm{LB}}^{B \& C}}$ is $-1.06 \%$. We note that for the 3 instances where computational time reached 3 hours with Benders Decomposition (S-CP, M-CP and M-RP), both lower and upper bounds are far from the lower bounds given by B\&C.

A second set of instances named Set 2 is generated based on the instance M-CP. This instance has been chosen because of its characteristics which are similar to the ones of the French local associations. This set of instances permit to test the influence of the number of hubs and maximal open hubs on the solving methods. Hence 6 configurations are proposed in Table 4. Starting from the configuration with the minimum number of hubs, the other ones are generated by inserting only additional new hub locations. The farmers and clients have the same locations in all the configurations, and the demands and costs are the same as well. This set of instances should also permit to evaluate the solving methods when the constraints on the restriction on the number of transshipments (constraints (3.16) and (3.17)) have an influence on the solution. Because of the cost structure, transshipment is expensive since the variable cost $c_{\mathrm{hi}}$ is paid for each transshipment. Hence we propose to limit the scope of actions of the hubs in order to favour the use of transshipment. For each configuration, three instances are built with a scope of actions for hubs $d^{\max } \in\{25 ; 35 ; \infty\}$. This second set of instances has 18 instances with parameters $H^{\max },|H|$ and $d^{\max }$ named $\mathrm{M}-\mathrm{CP}_{H^{\max }}^{|H|}-d^{\max }$ from Table 5.

Processor, memory and stopping criterion are the same as for Set 1 , with only a $2 \%$ gap as stopping criterion for the B\&C method. CPU time results are presented in Table 5.

Results show that with B\&C the CPU time is decreasing with the scope of action of the hubs, except for instances with maximal number of open hubs $H^{\max }=5$. Benders Decomposition is not competitive for these instances since the stopping criterion of 3 hours CPU time is always reached before the $5 \%$ gap stopping criterion. In the results of Set 1, M-CP was one of the instances for which results of Benders Decomposition were not good. The computational time of DSSP is not so good neither for two reasons. First, introducing scope of actions will not reduce significantly the computational time since there is the same number of combination of open hubs to evaluate. Secondly, increasing the number of hubs or the maximal number of hubs to open increases the computational time since there are more combinations of open hubs to evaluate. Hence the computation of a subset of combinations of hubs to open remains a good prospect for decreasing the computational time of the DSSP method.

Results about the gap with the lower bound of the B\&C are presented in Table 6 , and more detailed results with the lower bounds of the Benders Decomposition 
TABle 5. CPU time (in seconds) for Set 2.

\begin{tabular}{lccc}
\hline Instance & $\begin{array}{c}\text { B\&C } \\
(\text { CPLEX 2\% })\end{array}$ & $\begin{array}{c}\text { Benders } \\
\text { decomposition }(5 \%)\end{array}$ & DSSP \\
\hline $\mathrm{M}-\mathrm{CP}_{2}^{5}-\infty$ & 2317.50 & 11103.99 & 1877.64 \\
$\mathrm{M}-\mathrm{CP}_{2}^{5}-35$ & 449.69 & 10812.16 & 2379.74 \\
$\mathrm{M}-\mathrm{CP}_{2}^{5}-25$ & 147.16 & 10848.92 & 2648.68 \\
${\mathrm{M}-C P_{3}^{5}-\infty}^{5}$ & 790.74 & 10829.28 & 2211.89 \\
$\mathrm{M}-\mathrm{CP}_{3}^{5}-35$ & 554.41 & 10949.07 & 1692.73 \\
$\mathrm{M}-\mathrm{CP}_{3}^{5}-25$ & 302.94 & 10815.23 & 2769.15 \\
$\mathrm{M}-\mathrm{CP}_{3}^{7}-\infty$ & 2092.43 & 10908.02 & 8742.84 \\
$\mathrm{M}-\mathrm{CP}_{3}^{7}-35$ & 1077.80 & 10900.69 & 9619.28 \\
$\mathrm{M}-\mathrm{CP}_{3}^{7}-25$ & 362.69 & 11083.41 & 10340.01 \\
$\mathrm{M}-\mathrm{CP}_{5}^{7}-\infty$ & 515.01 & 11063.83 & 11804.77 \\
$\mathrm{M}-\mathrm{CP}_{5}^{7}-35$ & 1114.08 & 11559.28 & 6605.28 \\
$\mathrm{M}-\mathrm{CP}_{5}^{7}-25$ & 2602.05 & 10861.30 & 6726.62 \\
$\mathrm{M}-\mathrm{CP}_{3}^{10}-\infty$ & 4135.85 & 10875.44 & 33820.94 \\
$\mathrm{M}-\mathrm{CP}_{3}^{10}-35$ & 2971.53 & 11635.56 & 37133.93 \\
$\mathrm{M}-\mathrm{CP}_{3}^{10}-25$ & 1819.71 & 11618.24 & 41353.47 \\
$\mathrm{M}-\mathrm{CP}_{5}^{10}-\infty$ & 1315.07 & 10852.35 & 89766.92 \\
$\mathrm{M}-\mathrm{CP}_{5}^{10}-35$ & 2855.53 & 10952.36 & 112424.00 \\
$\mathrm{M}-\mathrm{CP}_{5}^{10}-25$ & 2050.98 & 11207.96 & 105775.99 \\
\hline
\end{tabular}

are presented in Table 8 of the Appendix. Again, these results show the very good quality of the DSSP method. The solutions of DSSP are better than the solutions given by CPLEX for 17 instances out of 18 . So the high computational time of DSSP is balanced with the very good solutions given by this method. For Benders Decomposition, when there are no scope of actions for the hubs, the solutions are quite good (but not as good as with the B\&C). When a scope of actions is considered, and transshipments between hubs are used, upper and lower bounds are of poor quality. Benders Decomposition is not really efficient for these instances, and should be improved adding cuts in the master problem in order to capture the flow between hubs in the sub-problem.

Hence these experimental results show the pros and cons of Benders Decomposition and DSSP when compared to B\&C using CPLEX in solving the SND-SLSC problem. The solving time can be very short with Benders Decomposition, at least for Set 1 except 3 instances. The solutions are of high quality with DSSP. Furthermore, both methods have the advantage of using a decomposition of the problem (by periods and by products), hence to require less memory space, specially for DSSP which only solves LP without any branching tree.

\section{Conclusion And PROSPeCts}

This paper has proposed a study on the service network design for short and local fresh food supply chain. The problem has original constraints: the number of transshipments for a product is restricted. A Mixed Integer Programming 
TABLE 6. Gap with CPLEX lower bound (in \%) for Set 2.

\begin{tabular}{|c|c|c|c|}
\hline Instance & $\begin{array}{c}\text { B\&C } \\
(\text { CPLEX 2\%) }\end{array}$ & $\begin{array}{c}\text { Benders } \\
\text { Decomposition }(5 \%)\end{array}$ & DSSP \\
\hline $\mathrm{M}-\mathrm{CP}_{2}^{5}-\infty$ & 1.20 & 3.23 & 0.60 \\
\hline $\mathrm{M}-\mathrm{CP}_{2}^{5}-35$ & 0.31 & 63.59 & 0.16 \\
\hline $\mathrm{M}-\mathrm{CP}_{2}^{5}-25$ & 0.07 & 52.09 & 0.04 \\
\hline $\mathrm{M}-\mathrm{CP}_{3}^{5}-\infty$ & 1.44 & 3.46 & 0.62 \\
\hline $\mathrm{M}-\mathrm{CP}_{3}^{5}-35$ & 0.44 & 90.68 & 0.23 \\
\hline $\mathrm{M}-\mathrm{CP}_{3}^{5}-25$ & 0.16 & 66.59 & 0.08 \\
\hline $\mathrm{M}-\mathrm{CP}_{3}^{7}-\infty$ & 1.30 & 3.48 & 0.62 \\
\hline $\mathrm{M}-\mathrm{CP}_{3}^{7}-35$ & 0.48 & 86.42 & 0.23 \\
\hline $\mathrm{M}-\mathrm{CP}_{3}^{7}-25$ & 0.15 & 72.71 & 0.09 \\
\hline $\mathrm{M}-\mathrm{CP}_{5}^{7}-\infty$ & 1.01 & 3.59 & 0.66 \\
\hline $\mathrm{M}-\mathrm{CP}_{5}^{7}-35$ & 0.45 & 88.17 & 0.29 \\
\hline $\mathrm{M}-\mathrm{CP}_{5}^{7}-25$ & 0.09 & 66.88 & 0.13 \\
\hline $\mathrm{M}-\mathrm{CP}_{3}^{10}-\infty$ & 1.29 & 3.48 & 0.62 \\
\hline $\mathrm{M}-\mathrm{CP}_{3}^{10}-35$ & 0.44 & 80.83 & 0.23 \\
\hline $\mathrm{M}-\mathrm{CP}_{3}^{10}-25$ & 0.19 & 83.28 & 0.10 \\
\hline$M-C P_{5}^{10}-\infty$ & 0.92 & 3.60 & 0.67 \\
\hline $\mathrm{M}-\mathrm{CP}_{5}^{10}-35$ & 0.43 & 84.08 & 0.27 \\
\hline $\mathrm{M}-\mathrm{CP}_{5}^{10}-25$ & 0.46 & 84.76 & 0.22 \\
\hline
\end{tabular}

formulation has been proposed. In order to efficiently solve this problem, we have proposed to adapt two approaches used for multi-commodity flow problems with fixed costs: Benders Decomposition and Dynamic Slope Scaling Procedure (DSSP). Experimental results have been conducted with several instance structures. The results show the relevance of the proposed methods to tackle large-scale instances of the problem. However, the methods can still be improved both in terms of solving time for DSSP and Benders Decomposition, and in terms of solution quality for Benders Decomposition.

Hence, the first prospect is to strengthen the two proposed approaches, by adding other constraints in the master problem of the Benders Decomposition, and by combining the DSSP methods with heuristic techniques to reduce the subsets of open hubs. An other prospect is to conduct other experiments with larger size instances, where the use of Branch and Cut would become an issue because of the limitation of memory space. 


\section{ApPENDICES}

TABLE 7. Detailed results for Set 1.

\begin{tabular}{|c|c|c|c|c|c|}
\hline \multirow{2}{*}{ Instance } & \multicolumn{2}{|c|}{ B\&C (CPLEX 2\%) } & \multicolumn{2}{|c|}{ Benders Decomposition (5\%) } & \multirow{2}{*}{$\begin{array}{c}\text { DSSP } \\
\text { UB }\end{array}$} \\
\hline & LB & UB & $\mathrm{LB}$ & UB & \\
\hline S-CP & 1238254 & 1253911 & 1208748 & 1289362 & 1248364 \\
\hline $\mathrm{S}-\mathrm{CM}$ & 2060689 & 2079202 & 2033832 & 2112495 & 2070739 \\
\hline S-RP & 2543262 & 2565079 & 2505223 & 2586700 & 2556741 \\
\hline S-RM & 1884738 & 1905152 & 1857062 & 1936416 & 1895418 \\
\hline $\mathrm{M}-\mathrm{CP}$ & 5362040 & 5417131 & 5230614 & 5563482 & 5397545 \\
\hline $\mathrm{M}-\mathrm{CM}$ & 8701466 & 8799556 & 8604862 & 8956347 & 8747667 \\
\hline M-RP & 7683182 & 7762708 & 7545125 & 7931132 & 7725420 \\
\hline M-RM & 7311774 & 7415003 & 7265418 & 7624619 & 7357353 \\
\hline L-CP & 824676000 & 824774000 & 824464338 & 825153059 & 824730379 \\
\hline $\mathrm{L}-\mathrm{CM}$ & 832606000 & 832751000 & 832525556 & 852194941 & 832691191 \\
\hline L-RP & 825024000 & 825127000 & 824784920 & 825298010 & 825099027 \\
\hline L-RM & 822827000 & 822977000 & 822683749 & 830893689 & 822918689 \\
\hline
\end{tabular}

TABLE 8. Detailed results for Set 2.

\begin{tabular}{|c|c|c|c|c|c|}
\hline \multirow{2}{*}{ Instance } & \multicolumn{2}{|c|}{ B\&C (CPLEX 2\%) } & \multicolumn{2}{|c|}{ Benders Decomposition (5\%) } & \multirow{2}{*}{$\begin{array}{c}\text { DSSP } \\
\text { UB }\end{array}$} \\
\hline & LB & $\mathrm{UB}$ & $\mathrm{LB}$ & UB & \\
\hline $12-\infty$ & 5355283 & 5419476 & 5212069 & 5534151 & 5387785 \\
\hline $\mathrm{P}_{2}^{5}-35$ & 363000 & 24439300 & 17322439 & 66911945 & 24402971 \\
\hline $\mathrm{P}_{2}^{5}-25$ & 772400 & 88833400 & 37829139 & 185297605 & 88804206 \\
\hline $\mathrm{CP}_{3}^{5}-\infty$ & 5353213 & 5430303 & 5210202 & 5545172 & 5386705 \\
\hline $\mathrm{CP}_{3}^{5}-35$ & 17501500 & 17578100 & 17282646 & 187847317 & 17541190 \\
\hline $3-25$ & 45362800 & 45437000 & 35153774 & 135795283 & 45401359 \\
\hline${ }_{3}^{7}-\infty$ & 5352409 & 54221 & 5209437 & 5545172 & 5385875 \\
\hline${ }_{3}^{7}-35$ & 17501600 & 17584800 & 17276430 & 128836106 & 17541190 \\
\hline $\mathrm{CP}_{3}^{7}-25$ & 45362600 & 45431200 & 35140143 & 166235633 & 45401359 \\
\hline $\mathrm{P}_{5}^{7}-\infty$ & 5352042 & 5406364 & 5208813 & 5551352 & 5387707 \\
\hline $\mathrm{M}-\mathrm{CP}_{5}^{7}-35$ & 17496900 & 17575200 & 17278499 & 147905786 & 17548079 \\
\hline $5-25$ & 35357100 & 35388600 & 35138921 & 106747795 & 35403961 \\
\hline $\mathrm{CP}_{3}^{10}-\infty$ & 53 & 5421109 & 5209129 & 5545172 & 5385875 \\
\hline $\mathrm{M}-\mathrm{CP}_{3}^{10}-35$ & 175 & 17578700 & 17275172 & 91280422 & 17541190 \\
\hline $\mathrm{M}-\mathrm{CP}_{3}^{10}-25$ & 40390800 & 40468000 & 20733170 & 241582674 & 40430851 \\
\hline $\mathrm{M}-\mathrm{CP}_{5}^{10}-\infty$ & 5351538 & 5400697 & 5208507 & 5551352 & 5387707 \\
\hline $\mathrm{M}-\mathrm{CP}_{5}^{10}-35$ & 17496600 & 17572100 & 17274953 & 109888501 & 17544329 \\
\hline $\mathrm{M}-\mathrm{CP}_{5}^{10}-25$ & 20974300 & 21070000 & 20731817 & 137642051 & 21021367 \\
\hline
\end{tabular}




\section{REFERENCES}

[1] R.K. Ahuja, T.L. Magnanti and J.B. Orlin, Network Flows: Theory, Algorithms, and Applications. Prentice Hall, Englewood Cliffs, NJ (1993).

[2] C. Barnhart, N. Krishnan, D. Kim and K. Ware, Network design for express shipment delivery. Comput. Opt. Appl. 21 (2002) 239-262.

[3] J.F. Benders, Partitioning Procedures for Solving Mixed-Variables Programming Problems. Numer. Math. 4 (1962) 238-252.

[4] A.M. Costa, A survey on benders decomposition applied to fixed-charge network design problems. Comput. Oper. Res. 32 (2005) 1429-1450.

[5] T.G. Crainic, Service network design in freight transportation. Eur. J. Oper. Res. 122 (2000) 272-288.

[6] T.G. Crainic and G. Laporte, Planning models for freight transportation. Eur. J. Oper. Res. 97 (1997) 409-438.

[7] B. Gendron, Decomposition methods for network design. Procedia - Social Behavioral Sci. 20 (2011) 31-37.

[8] G. Guisewite and P. Pardalos, Minimum concave-cost network flow problems: Applications, complexity, and algorithms. Annal. Oper. Res. 25 (1990) 75-99.

[9] D. Kim and P.M. Pardalos, A solution approach to the fixed charge network flow problem using a dynamic slope scaling procedure. Oper. Res. Lett. 24 (1999) 195-203.

[10] N. Megiddo and K.J. Supowit, On the complexity of some common geometric location problems. SIAM J. Comput. 13 (1984) 182-196.

[11] S. Melkote and M.S. Daskin, Capacitated facility location/network design problems. Eur. J. Oper. Res. 129 (2001) 481-495.

[12] F. Ortega and L.A. Wolsey, A branch-and-cut algorithm for the single-commodity, uncapacitated, fixed-charge network flow problem. Networks 41 (2003) 143-158.

[13] S.H. Owen and M.S. Daskin, Strategic facility location: A review. Eur. J. Oper. Res. 111 (1998) 423-447.

[14] E. Schlich and U. Fleissner, The ecology of scale: Assessment of regional energy turnover and comparison with global food. Int. J. Life Cycle Assessment 10 (2005) 219-223.

[15] N. Teypaz, S. Schrenk and V.-D. Cung, A decomposition scheme for large-scale Service Network Design with asset management. Transp. Res. Part E 46 (2009) 156-170.

[16] N. Wieberneit, Service network design for freight transportation: a review. OR Spectrum 30 (2008) $77-112$. 